\title{
Intramedullary hemangioblastomas: surgical results in 16 patients
}

\author{
Andrei F. Joaquim, MD, PhD, ${ }^{1}$ Enrico Ghizoni, MD, PhD, ${ }^{1}$ Marcos Juliano dos Santos, MD, ${ }^{1}$ \\ Marcelo Gomes C. Valadares, MD, ${ }^{1}$ Felipe Soares da Silva, ${ }^{2}$ and Helder Tedeschi, MD, PhD' \\ ${ }^{1}$ Neurosurgery Division, ${ }^{2}$ State University of Campinas (UNICAMP), Campinas, São Paulo, Brazil
}

\begin{abstract}
OBJECT Hemangioblastomas are rare, benign, highly vascularized tumors that can be found throughout the neuraxis but are mainly located in the cerebellum and in the spinal cord. Spinal hemangioblastomas can present with motor and sensory deficits, whose severity varies according to the size and location of the tumor. Resection is the best treatment option to avoid neurological deterioration. The authors report surgical results in the treatment of intramedullary hemangioblastomas and discuss the technical nuances important to achieving total resection without adding new deficits.
\end{abstract}

METHODS A consecutive series of patients with intramedullary hemangioblastomas operated on between 2000 and 2014 by the senior author (H.T.) is presented. The functional scale proposed by McCormick was used to evaluate the patients' neurological status before and after surgery.

RESULTS Sixteen patients were included in the study and underwent 17 surgeries. Follow-up was at least 6 months. Age at presentation varied from 13 to 58 years (mean 33.8 years). Ten patients (62.5\%) were males and 6 patients $(37.5 \%)$ were females. Seven $(43.75 \%)$ of the 16 patients had associated von Hippel-Lindau syndrome, with hemangioblastomas also presenting in other locations. Three patients had multiple tumors in the same segment in the spinal cord, and 10 patients (62.5\%) presented with cysts. According to the site of presentation, 11 tumors (68.75\%) were localized at the cervical region (including the cervicomedullary junction) and 5 tumors (31.25\%) at the thoracic level. Total resection was achieved in all cases, evidenced by postoperative MRI. Four patients had some functional worsening immediately after surgery. After 6 months, 1 patient had functional worsening compared with preoperative status, and 2 patients had clinical improvement. The majority of the patients remained clinically stable postoperatively.

CONCLUSIONS Adequate knowledge of anatomy and the correct use of microsurgical techniques allowed total resection of these tumors, with minimal morbidity and maximum functional recovery. Outcome seems to be directly related to the neurological status before surgery.

http://thejns.org/doi/abs/10.3171/2015.5.FOCUS15171

KEY WORDS hemangioblastomas; tumor; intramedullary; diagnosis; surgery; treatment

$\mathrm{H}$ EMANGIOBLASTOMAS can be found throughout the neuraxis, but the most common sites of occurrence in the CNS are the cerebellum and the spinal cord. ${ }^{2-4,7}$ They are rare, benign, highly vascularized tumors classified as Grade I according to the World Health Organization classification system. ${ }^{2,7}$ About $3 \%$ of all the intramedullary tumors are hemangioblastomas. Hemangioblastomas occur as sporadic lesions in about $70 \%-80 \%$ of cases, whereas in 20\%-30\% of cases they can be secondary to a dominantly inherited genetic familial cancer syndrome known as von Hippel-Lindau (VHL) syndrome. ${ }^{4,7,11}$
This rare genetic disorder is characterized by multiple hemangioblastomas in the retina and in the central nervous system as well as visceral cysts, especially in the pancreas and kidneys, with an increased risk for malignant transformation into carcinomas..$^{89,12}$ The mutation responsible for the VHL syndrome is located in the chromosome 3p25-26, also known as the $V H L$ gene, a tumor suppressor gene that also can be found in some of the sporadic hemangioblastomas. ${ }^{1,8,9,12}$ Tumors in patients with VHL syndrome require continuous clinical and radiological monitoring due to the high potential for fast tumor growth. In patients with VHL

ABBREVIATIONS AVM = arteriovenous malformation; ICU = intensive care unit; VHL = von Hippel-Lindau.

SUBMITTED March 31, 2015. ACCEPTED May 20, 2015.

INCLUDE WHEN CITING DOI: 10.3171/2015.5.FOCUS15171.

DISCLOSURE The authors report that they have received no funds in support of this study, that they have no financial interest in the subject of this article, that no benefits in any form have been or will be received from a commercial party related directly or indirectly to the subject of this manuscript, and that the manuscript submitted does not contain information about medical device(s). 
syndrome, multiple asymptomatic tumors are usually just followed to avoid an excessive number of surgical interventions. . $^{72,16}$

Clinical presentation depends on the size and location of the tumor. The presence of tumoral cysts, a common characteristic of hemangioblastomas, can also be responsible for neurological deterioration. Motor and sensory deficits can be present and vary from mild paresthesias to severe paralysis. Once the neurological symptoms appear, resection should be attempted to avoid further deterioration. $7,15,16$

Considering the rarity of these tumors, we report our surgical results in the treatment of intramedullary hemangioblastomas and discuss important technical nuances necessary to achieve total resection and to avoid adding new neurological deficits.

\section{Methods}

Patients surgically treated for intramedullary hemangioblastomas operated on between 2000 and 2014 by the senior author (H.T.) were retrospectively reviewed and included in this study. Institutional review board approval was obtained as part of our project.

The functional scale proposed by McCormick was used to evaluate the patients' neurological status before and after surgical treatment (Table 1). ${ }^{10}$ Patients were screened for VHL syndrome in the setting of multiple tumors. Postoperative complications were described in detail.

\section{Surgical Technique}

During the induction of anesthesia, dexamethasone was administered intravenously at an initial $10 \mathrm{mg}$, followed by $4 \mathrm{mg}$ every 6 hours. Antibiotics were also prescribed. Neurophysiological monitoring of sensory and motor evoked potentials is useful and was used in the last 7 patients of our series.

Patients were operated on in ventral decubitus position, with an en bloc, nonexpandable laminoplasty; the laminae were exposed from the superior to the inferior margin of the tumor according to the MRI. Patients with tumors above the T-2 level had their head supported in a head fixation system. Patients with craniocervical tumors also underwent a posterior fossa craniotomy. After laminoplasty, the dura mater was opened along the midline and anchored with Prolene 4-0 sutures to avoid bleeding from the extradural space into the spinal cord canal. Under microscopic magnification, the arachnoid was dissected, and the surface of the tumor was fully exposed. Surgery was performed in a way similar to that employed for an arteriovenous malformation (AVM) of the CNS. On the surface of the cord, the afferent vessels to the tumor are meticulously coagulated. Coagulation should be low intensity and carried out close to the tumor to avoid damage to the spinal cord. The thick pia-arachnoid around the tumor that involves the cord is then carefully cut, and a dissection plane between the tumor and the cord is developed. When present, tumoral cysts can be helpful during dissection (Fig. 1), as they tend to naturally isolate parts of the tumor from the surrounding nervous tissue. No attempts at debulking the tumor should be made as it could result in uncontrollable bleeding. Major draining veins should be left intact throughout the operation. Lastly, after coagulation of all afferent vessels and dissection of the tumor, the major veins are coagulated and the tumor is removed. Figures 2 and 3 illustrate our surgical technique. Reconstruction is carried out with the laminae being fixed using nylon sutures or titanium miniplates (Fig. 4).

After surgery, our general protocol includes postoperative Day 1 in the intensive care unit (ICU), followed by 2 or 3 days in the general ward with early ambulation (when feasible) and early rehabilitation with physical therapy.

\section{Results}

Sixteen patients, with a total of 17 surgeries, were operated on and included in this study. Ages ranged from 13 to 58 years (mean 33.8 years). Ten of our patients (62.5\%) were males and 6 patients $(37.5 \%)$ were females. Seven $(43.75 \%)$ of the 16 patients had VHL syndrome. The mean follow-up time was 48 months (range 6 months-12 years). Table 2 summarizes the clinical data for the 16 patients who underwent surgery.

One patient was operated on twice, first at 13 years for cervical decompression ( 3 hemangioblastomas) and then at 18 years for a thoracic tumor, with a total of 4 tumors. Another 2 patients presented with multiple different size lesions at the cervical level. One of the patients presented with multiple associated cysts (Fig. 5) and had 8 of her lesions surgically removed (2 tumors $>1 \mathrm{~cm}$ and 6 tumors $<5 \mathrm{~mm}$ in size). The other patient had no cysts but had 12 tumors of various sizes removed ( 1 tumor $>1 \mathrm{~cm}$ and 11 tumors $\leq 5 \mathrm{~mm}$ ). A total of 37 hemangioblastomas were operated on in the 16 patients. Cerebellar lesions were present in $5(71 \%)$ of the 7 patients with VHL syndrome. Except for Case 14, all cerebellar lesions were also operated on by the senior author (H.T.). They were operated on during the same procedure whenever possible, with the symptomatic lesion being operated on first.

TABLE 1. Clinical/functional classification scheme*

\begin{tabular}{cc}
\hline Grade & Definition \\
\hline I & Neurologically normal; mild focal deficit not significantly affecting function of involved limb; mild spasticity or reflex abnormality; normal gait \\
\hline II & $\begin{array}{c}\text { Presence of sensorimotor deficit affecting function of involved limb; mild to moderate gait difficulty; severe pain or dysesthetic syndrome } \\
\text { impairing patient's quality of life; still functions \& ambulates independently }\end{array}$ \\
\hline III & $\begin{array}{c}\text { More severe neurological deficit; requires cane/brace for ambulation or significant bilateral upper extremity impairment; may or may not func- } \\
\text { tion independently }\end{array}$ \\
\hline IV & Severe deficit; requires wheelchair or cane/brace w/ bilateral upper extremity impairment; usually not independent \\
\hline
\end{tabular}

\footnotetext{
* McCormick Scale reproduced with permission from McCormick et al.: J Neurosurg 72:523-532, 1990.
} 


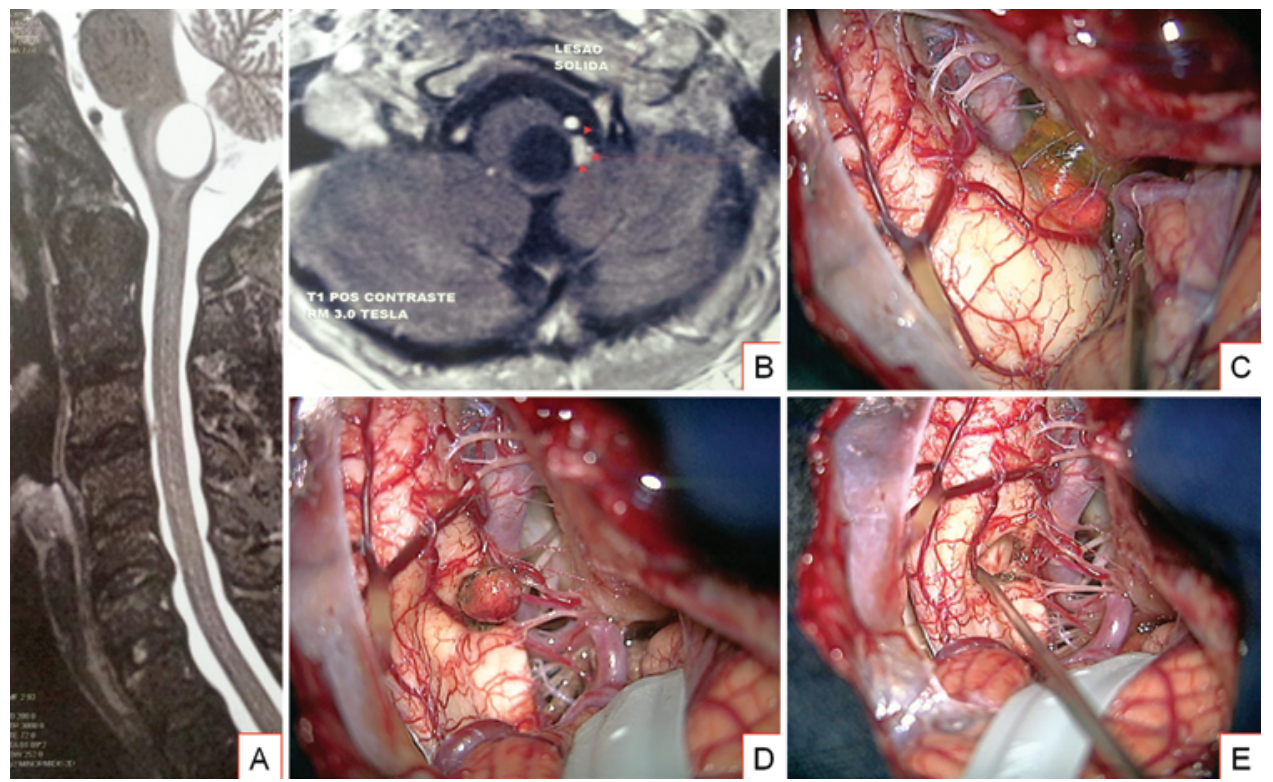

FIG. 1. Case 11. A: Sagittal T2-weighted MR image sequence showing a cyst in the medulla. B: Axial T1-weighted MR image with contrast enhancement showing a nodule in the lateral portion of the cyst (arrows), suggestive of a tumor. C: Intraoperative photograph of the lesion after a left far-lateral exposure. The lesion covered by a cyst can be seen on the surface of the cord, which is bloated with the intramedullary component of the cyst. D: The tumor has been dissected and is shown lying on the surface of the cord. The cystic cavity has been entered, and the cord has become flattened after the cyst fluid was drained. E: The cystic cavity is inspected after the tumor has been removed.

Regarding tumor location, $11(68.75 \%)$ were localized at the cervical region (including 4 tumors in the cervicomedullary junction), and $5(31.25 \%)$ were situated at the thoracic spinal cord. Three patients had single tumors located off the midline, and 3 patients had tumors in multiple locations in the cord. All the remaining patients had midline lesions. In Fig. 6, we present an illustration of a cervicomedullary tumor surgery.

Considering early neurological outcome, assessed just before hospital discharge, 4 patients worsened, scoring 1 grade lower on the McCormick Scale, whereas 10 patients maintained the same status they had before surgery, and 1 patient had an early improvement, scoring 1 grade higher (from III to II). Transitory neuropathic pain was a common finding $(70 \%)$ and usually subsided in a couple of weeks. Two patients had persistent neuropathic pain after surgery that required continuous use of pain medication. One of these patients complained after surgery of "electric shocks" that would run down his back and into his limbs whenever he would bend his neck or stretch his back (Lhermitte's sign). One patient had a hemiballism of her left arm preoperatively (caused by impairment of proprioception due to a tumor located off the midline in the cervical region) that has shown only little improvement after surgery. Twelve patients were previously normally ambulatory, and of these, 7 patients complained of some difficulty in walking during the first 2-3 weeks after surgery, probably due to transitory impairment of the dorsal columns. All patients who had ambulatory problems postoperatively had midline lesions and had been operated on through a posterior myelotomy. Difficulty in ambulation was slightly more prevalent in those patients with thoracic lesions (4 patients) than in those with cervical lesions (3
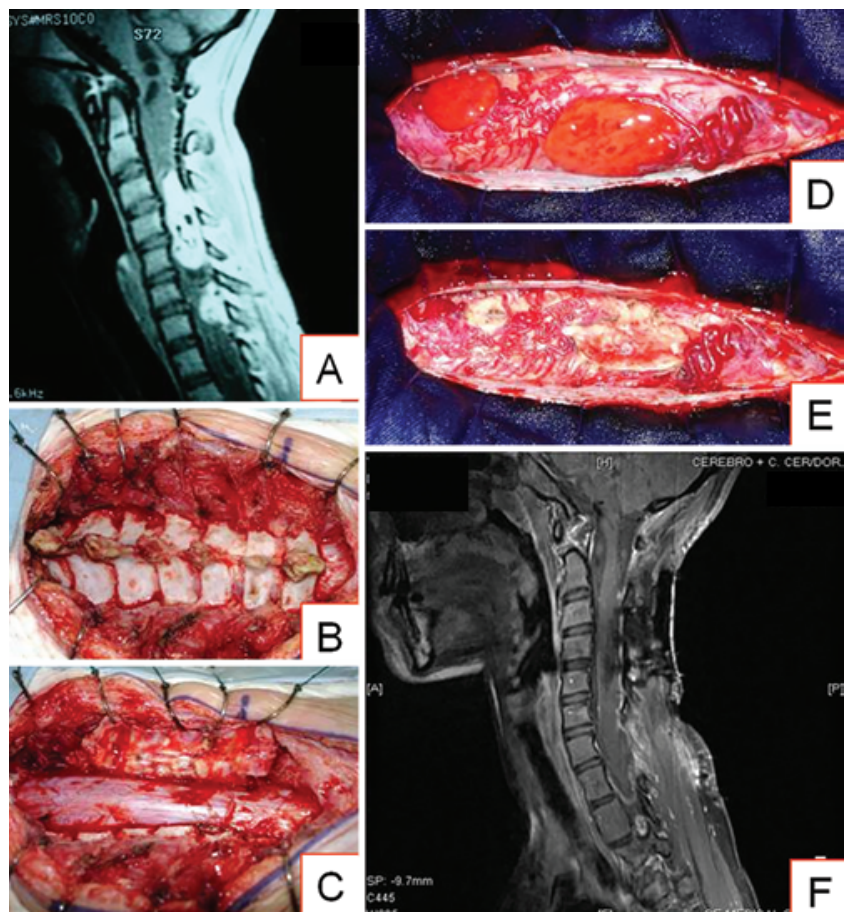

FIG. 2. Case 1. A: Preoperative sagittal T1-weighted MR image with contrast showing 2 intramedullary lesions at C2-4 and C-6. B: Intraoperative photograph showing exposure of the laminae from C-2 to T-1. C: Photograph of the intraoperative view after the (open door) laminoplasty was performed. D: Intraoperative view showing the hemangioblastomas after dura mater opening. E: Photograph of the final aspect of the spinal cord after tumor resection. F: Postoperative cervical sagittal T1-weighted MR image with contrast. Reprinted from Joaquim et al: Surgical management of intramedullary hemangioblastomas. Coluna/Columna 8: 274-278, 2009. Published with permission. 


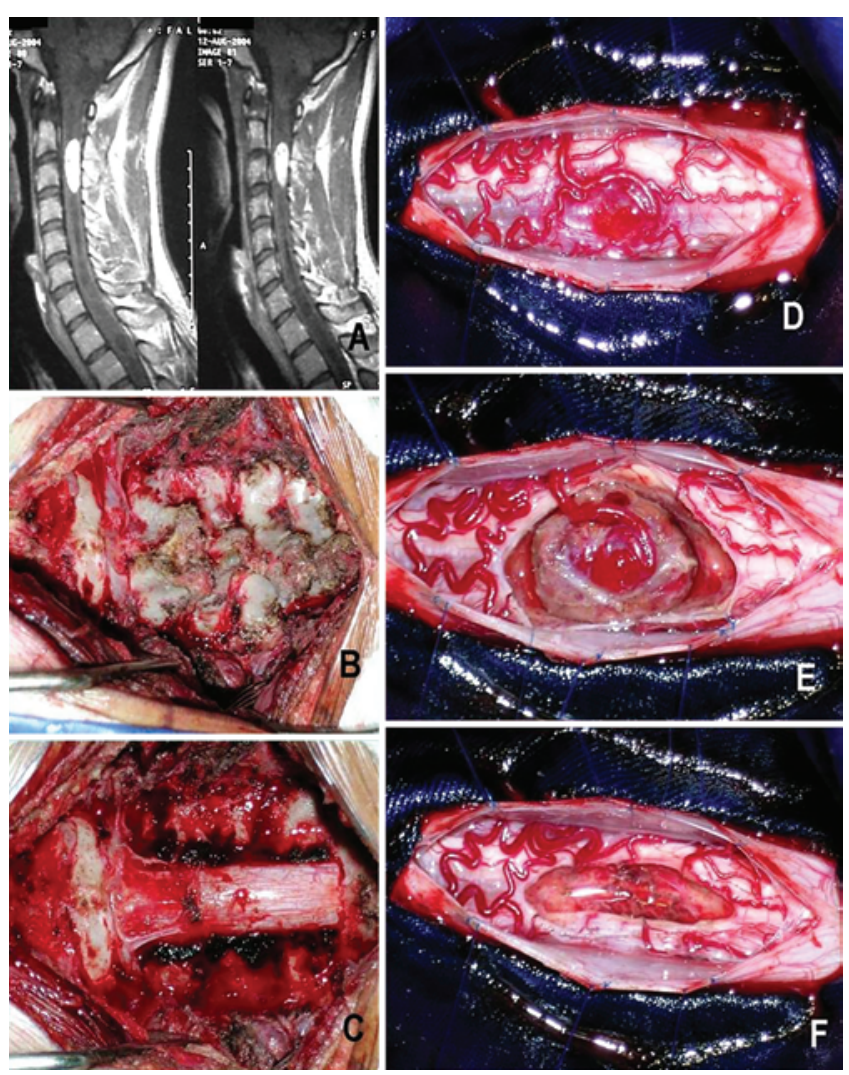

FIG. 3. Case 2. A: Preoperative sagittal T1-weighted MR image showing an intramedullary tumor from C-2 to C-4. B: Intraoperative photograph showing an exposure from the occiput to $\mathrm{C}-5$ was performed with removal of the posterior arch of C-1. C: Intraoperative view after (open door) laminoplasty from C-2 to C-5. D: Photograph taken after dural opening showing the hemangioblastoma and its respective drainage vein. E: View showing microdissection of the tumor and maintenance of the drainage vein. F: Photograph of the final aspect of the spinal cord after tumor resection. Reprinted from Joaquim et al: Surgical management of intramedullary hemangioblastomas. Coluna/Columna 8: 274-278, 2009. Published with permission.

patients). In general, all patients managed to walk freely on the following postoperative day in the hospital with the aid of a large-base cane and received early rehabilitation. At 3 months, only 1 of the patients (Case 10) still required assistance to walk. In regard to ambulation in the remaining 4 patients, 1 patient was restricted to a wheelchair, and 3 patients were able to stand up with the aid of crutches and manage to do short walks preoperatively. Their ability to walk has not been changed by surgery.

One patient (Case 14) stayed longer in the ICU due to a severely impaired preoperative pulmonary capacity that demanded prolonged ventilatory support but had no infections. Another patient (Case 10) had a preoperative neurogenic bladder and developed a urinary tract infection postoperatively due to prolonged vesical catheterization. He was discharged on the 14th postoperative day with no improvement in bladder function. The average length of stay in the hospital was 6.3 days (range 4-14 days). None of our patients required blood transfusions, nor did they have epidural hematomas or skin infections. There were no deaths in our series as well.
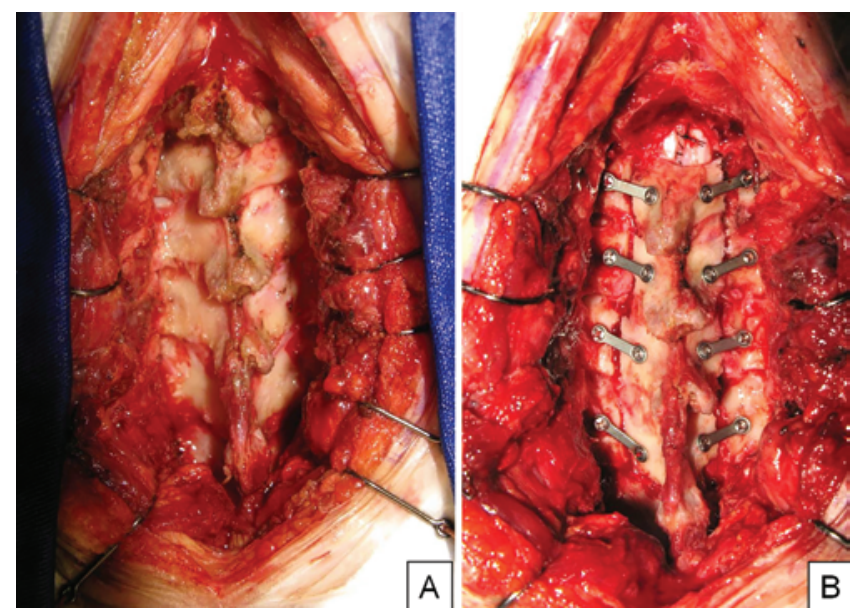

FIG. 4. Case 9. Intraoperative photographs of the laminoplasty technique. A: Laminae and the spinous processes of C3-6 are exposed. B: Intraoperative view after a C3-6 laminoplasty, showing the posterior elements reconstructed with titanium miniplates.

Considering late neurological outcome with at least 6 months of follow-up, 2 patients (12.5\%) improved their initial McCormick grade, 1 patient had a worsening $(6.25 \%)$ of 1 grade, and 14 patients $(81.25 \%)$ maintained the same neurological status.

Complete tumor resection was obtained in all patients.

\section{Discussion}

Hemangioblastomas are rare CNS tumors that can be found mainly in the cerebellum but may also be found in the spinal cord and brainstem. ${ }^{1,13,14}$ Clinical symptoms may vary according to tumor size, location, and the presence of concomitant cysts that may cause mass effect. ${ }^{7,13,14}$ Surgical treatment is well accepted in symptomatic patients, with some controversies regarding conservative management in asymptomatic lesions, especially in the setting of VHL syndrome. ${ }^{12,13}$

Most of the tumors arise in the dorsal or dorsolateral portion of the spinal cord and usually present initially with pain or other dysesthesias. When located in the dorsal portion of the spinal cord, surgical treatment tends to be less morbid, as the motor tracts are not transgressed during the approach. Tumors that arise in the anterior surface of the cord are usually not amenable to surgical treatment due to the risk of damage to the anterior spinal artery, which may result in devastating neurological deficits.

The association of hemangioblastomas and VHL increases the chances for multiple tumors. For this reason, all patients with a hemangioblastoma should be screened for other tumors in the entire CNS. In our series, all patients had a complete (whole neuraxis) workup with MRI, abdominal CT scanning, and eye funduscopy performed preoperatively.

Seven of our patients had VHL syndrome, and 2 patients underwent more than 1 surgery. The first was a young girl operated on at age 13 years (Case 1) and again at age 18 years, with uneventful recoveries in both procedures. The second patient had been operated on 3 times elsewhere and was referred to us because of recurring tu- 
TABLE 2. Clinical data of 16 patients who underwent surgical treatment of intramedullary hemangioblastomas

\begin{tabular}{|c|c|c|c|c|c|c|c|}
\hline \multirow[b]{2}{*}{ Case No. } & \multirow[b]{2}{*}{ Sex, Age (yrs) } & \multirow[b]{2}{*}{ Spinal Cord Site } & \multirow[b]{2}{*}{ VHL } & \multirow[b]{2}{*}{ Other CNS Tumors } & \multicolumn{3}{|c|}{ McCormick Grade* } \\
\hline & & & & & Preop & Postop & $\geq 6$ Mos After Surgery \\
\hline $1 \dagger$ & $F, 13$ & C & Yes & $\mathrm{T}$ & III & $\|$ & I \\
\hline & 18 & $\mathrm{~T}$ & & CT & ॥ & II & II \\
\hline $2 \dagger$ & $M, 45$ & C & Yes & CRBL & I & 1 & I \\
\hline 3 & $F, 23$ & C & No & - & I & II & I \\
\hline 4 & $F, 24$ & C & No & - & II & II & II \\
\hline 5 & M, 39 & $T$ & No & - & II & III & II \\
\hline 6 & $\mathrm{~F}, 20$ & $\mathrm{CM}$ & No & - & II & II & I \\
\hline 7 & $\mathrm{~F}, 17$ & $\mathrm{CM}$ & Yes & - & III & III & III \\
\hline 8 & M, 37 & $\mathrm{~T}$ & No & - & 1 & II & 1 \\
\hline 9 & $M, 26$ & C & No & - & II & II & II \\
\hline 10 & M, 52 & $\mathrm{~T}$ & Yes & CRBL & II & III & III \\
\hline 11 & M, 58 & $\mathrm{CM}$ & No & - & 1 & I & 1 \\
\hline 12 & $M, 55$ & $T$ & Yes & CRBL & IV & III & III \\
\hline 13 & M, 57 & $\mathrm{~T}$ & No & - & II & II & II \\
\hline $14 \dagger$ & $F, 33$ & C & Yes & CRBL, T & III & III & III \\
\hline 15 & M, 25 & CT & No & - & II & II & II \\
\hline 16 & M, 33 & $\mathrm{CM}$ & Yes & CRBL & II & II & II \\
\hline
\end{tabular}

$\mathrm{C}=$ cervical; $\mathrm{CM}=$ cervicomedullary; $\mathrm{CRBL}=$ cerebellum; $\mathrm{CT}=$ cervicothoracic $\mathrm{T}=$ thoracic.

* Neurological status outcome evaluated according to the scale proposed by McCormick et al. ${ }^{10}$

† Multiple tumors at the same spine segment.

mors in the cervical spine, with cystic formations throughout the entire spinal cord. Her preoperative neurological status was poor, and she showed no marked improvement postoperatively. Cases 1 and 14, mentioned earlier, are not enough to evaluate if repeated surgeries may indeed impact on neurological improvement and functional outcome of patients with VHL syndrome. Further analyses based on more substantial data are required.

The criteria for diagnosis of VHL disease is made based on familial history or on the presence of concomitant hemangioblastomas in the retina. ${ }^{13,15}$ In the absence of familial history, at least 2 hemangioblastomas in the CNS (including the retina) are necessary to establish the diagnosis, or 1 hemangioblastoma in the CNS and at least 1 of the following tumors: renal carcinoma, visceral cyst, or pheochromocytoma or an elucidation of a deleterious mutation in the VHL gene. ${ }^{13,15}$

Surgical treatment can be curative for patients with single lesions, but continuous follow-up is necessary due to the high incidence of mutations in the VHL gene in patients with these tumors and a potential risk of a new tumor. ${ }^{7,13,15}$ A meticulous surgical technique is mandatory; the surgeon should use low-intensity bipolar coagulation only on the afferent arteries and avoid venous occlusion before total tumor resection, which may lead to tumor ingurgitation and excessive bleeding.7 If several superficial veins participate in the drainage of the tumor, the surgeon should try to maintain their patency as long as possible during dissection of the tumor. When that is not possible, the largest vein should, at all costs, be kept intact until the end of the procedure (the same rule applies to tumors that have single draining veins). When dissection is completed, the vein should be coagulated and cut close to the surface of the tumor to avoid venous infarction of the normal cord. These highly vascularized tumors cannot be debulked, and should be operated on in the same fashion as for an AVM.

The spinal cord is a very narrow, uniformly organized structure, with vessels that can be easily identified and are readily available for control during surgery. Vessels that feed intramedullary hemangioblastomas are small, superficial, and terminate at the tumor. Thus, arterial feeders should be coagulated close to the surface of the tumor, along with the tiny perforating vessels that are encountered during the dissection. In contrast to AVMs, there are no "en passage" vessels nourishing the tumor. For the reasons mentioned above, we have not used preoperative angiography or 3D-CT angiography in any of our cases. An exception to that rule was applied to the only case not included in this study, where the patient presented with an anteriorly located C-5 tumor. To evaluate the anterior spinal artery involvement, the patient underwent a digital subtraction angiogram. The anterior spinal artery could not be well depicted at the level of the tumor, and surgery was precluded.

We also have not used preoperative embolization because arterial vessels that supply hemangioblastomas are usually very irregular and small, making catheterization and complete obliteration impossible. We find no place for partial embolization in the surgical strategy for hemangioblastomas.

In cases where the tumor does not reach the pial surface, a midline myelotomy must be performed. ${ }^{7}$ To minimize damage to the posterior columns, the incision should always be restricted to the midline. The dentate ligaments and the exit of the neural rootlets from the spinal cord on 


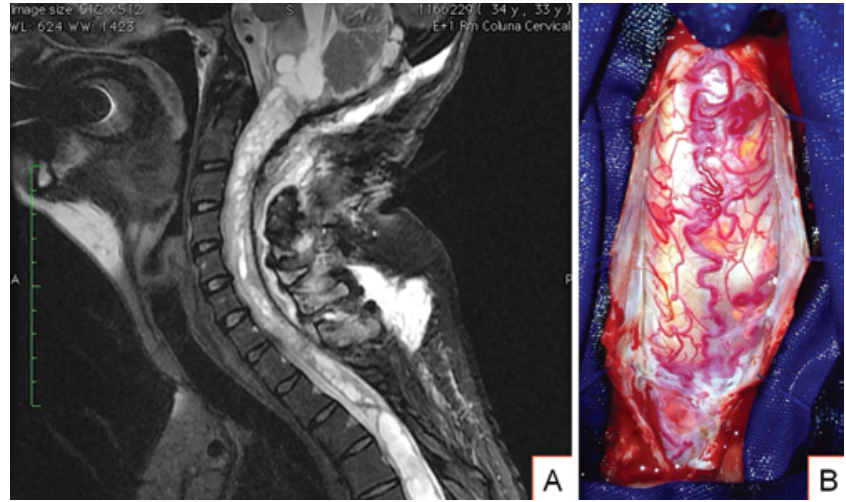

FIG. 5. Case 14. A: Cervical sagittal T2-weighted MR image obtained in a patient with VHL syndrome and multiple cystic lesions. B: Intraoperative photograph obtained in the same patient.

each side can serve as landmarks to the midline and guide the surgeon. ${ }^{5,7}$ Sutures are usually applied to the thick piaarachnoid on each side of the myelotomy after superficial dissection to gently aid in the retraction of the neural tissue that surrounds the tumor.

In our series, $81.25 \%$ of the patients remained neurologically stable, with no significant neurological improvement after surgery. This is similar to other series and suggests benefits in treating patients as soon as the diagnosis is made and before any neurological deterioration occurs. ${ }^{4,7,9}$ When effective, surgical treatment should be able to maintain the patients' previous neurological status.

Neuropathic pain and dysesthesias after surgery are consequences of disruption or manipulation of the posterior tracts necessary to approach the tumor. These symptoms were well managed with physical therapy and neuropathic drugs, such as gabapentin and pregabalin, and generally disappear in a matter of weeks.

Regarding postoperative spinal deformity, none of our patients developed cervical kyphosis after the laminoplasty that required surgery. Although there is no high level of evidence suggesting that laminoplasty is better than laminectomy, we strongly believe that laminoplasty can avoid postoperative deformity. ${ }^{6}$ Additionally, titanium miniplates are used to achieve immediate rigid fixation and to avoid potential complications of lamina migration and possible cord compression. Miniplates and miniscrews should be applied first to the lamina outside of the surgical cavity, and only then should one fixate it to the lateral masses so as to prevent inadvertent pressure on the spinal cord during screwing. Avoiding unnecessary violation of the facet joints and the removal of $\mathrm{C}-2$ and $\mathrm{C}-7$ spinous processes may help prevent postoperative deformity. ${ }^{6}$

None of our patients required any adjuvant postoperative treatment. The role of radiotherapy and chemotherapy in the management of these lesions remains controversial. ${ }^{2}$

Finally, although neurophysiological monitoring with sensory and motor evoked potentials is now part of our routine, in our first case series, preliminarily published in 2009, all patients were operated on without it and with comparable results. Neurophysiological monitoring serves well as a warning against neural damage. All changes detected during the procedure are carefully evaluated by the surgeon and the neurophysiologist. When potentials drop, the dissection stops, and the potentials are allowed to recover with warm saline irrigation of the spinal cord. There has never been a need to change the surgical technique during the procedure nor to bring the surgery to a complete halt due to changes in neurophysiological potentials. It is our strong opinion that the use of neurophysiological monitoring, accompanied with a meticulous surgical technique that avoids disruption of the spinal cord outside the tumor boundaries, can work almost as a guarantee that postoperative deficits will not occur.

The probable limitations of our study reside in the fact that it refers to a single surgeon's experience and is based on a relative small number of cases. However, considering the rarity of intramedullary hemangioblastomas and that the total number of tumors is much more expressive than the number of patients, we believe that our experience can be useful for surgeons who are not used to treating this challenging disease.

\section{Conclusions}

Total resection of intramedullary hemangioblastomas is the main treatment for these lesions. Surgery generally maintains the patients' preoperative neurological status
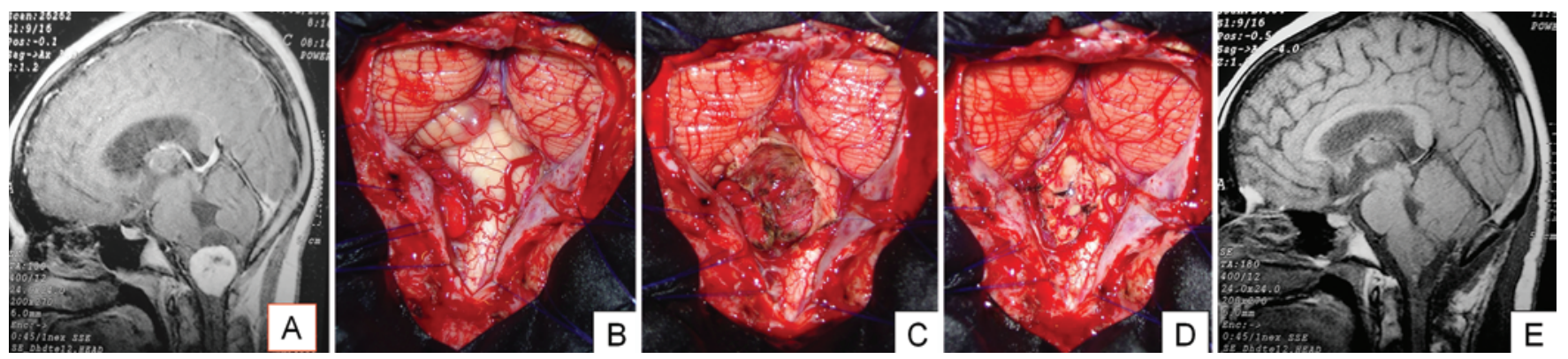

FIG. 6. Case 7. A: Preoperative sagittal T1-weighted MR image with contrast showing a cervicomedullary hemangioblastoma. B-D: Intraoperative views of the lesion with a midline suboccipital and upper cervical exposure. B: Photograph showing a lesion on the left lateral surface of the upper cervical cord, which is enlarged. Note a second lesion on the left cerebellar tonsil. C: Photograph taken after the posterior myelotomy has been performed, showing the lesion fully exposed. D: Photograph showing the cervicomedullary lesion completely removed, along with the small tonsillar tumor. E: Postoperative sagittal T1-weighted MR image showing complete resection of the hemangioblastoma. 
and thus should be indicated before their condition deteriorates. Screening for additional lesions is necessary because of the risk of VHL syndrome and multiple tumors. Diligent microsurgical techniques can help surgeons to improve their results in the treatment of patients with these rare lesions.

\section{References}

1. Catapano D, Muscarella LA, Guarnieri V, Zelante L, D'Angelo VA, D'Agruma L: Hemangioblastomas of central nervous system: molecular genetic analysis and clinical management. Neurosurgery 56:1215-1221, 2005

2. Chang SD, Meisel JA, Hancock SL, Martin DP, McManus M, Adler JR Jr: Treatment of hemangioblastomas in von HippelLindau disease with linear accelerator-based radiosurgery. Neurosurgery 43:28-35, 1998

3. Conway JE, Chou D, Clatterbuck RE, Brem H, Long DM, Rigamonti D: Hemangioblastomas of the central nervous system in von Hippel-Lindau syndrome and sporadic disease. Neurosurgery 48:55-63, 2001

4. Cristante L, Herrmann HD: Surgical management of intramedullary hemangioblastoma of the spinal cord. Acta Neurochir (Wien) 141:333-340, 1999

5. Joaquim AF, Almeida JP, Dos Santos MJ, Ghizoni E, de Oliveira E, Tedeschi H: Surgical management of intradural extramedullary tumors located anteriorly to the spinal cord. J Clin Neurosci 19:1150-1153, 2012

6. Joaquim AF, Cheng I, Patel AA: Postoperative spinal deformity after treatment of intracanal spine lesions. Spine J 12:1067-1074, 2012

7. Joaquim AF, Santos MJ, Tedeschi H: [Surgical management of intramedullary hemangioblastomas.] Coluna/Columna 8:274-278, 2009 (Portuguese)

8. Latif F, Tory K, Gnarra J, Yao M, Duh FM, Orcutt ML, et al: Identification of the von Hippel-Lindau disease tumor suppressor gene. Science 260:1317-1320, 1993

9. Lonser RR, Weil RJ, Wanebo JE, DeVroom HL, Oldfield EH: Surgical management of spinal cord hemangioblastomas in patients with von Hippel-Lindau disease. J Neurosurg 98:106-116, 2003
10. McCormick PC, Torres R, Post KD, Stein BM: Intramedullary ependymoma of the spinal cord. J Neurosurg 72:523532,1990

11. Melmon KL, Rosen SW: Lindau's disease: Review of the literature and study of a large kindred. Am J Med 36:595-617, 1964

12. Miller DJ, McCutcheon IE: Hemangioblastomas and other uncommon intramedullary tumors. J Neurooncol 47:253270,2000

13. Neumann HP, Eggert HR, Weigel K, Friedburg H, Wiestler OD, Schollmeyer P: Hemangioblastomas of the central nervous system. A 10 -year study with special reference to von Hippel-Lindau syndrome. J Neurosurg 70:24-30, 1989

14. Roonprapunt C, Silvera VM, Setton A, Freed D, Epstein FJ, Jallo GI: Surgical management of isolated hemangioblastomas of the spinal cord. Neurosurgery 49:321-328, 2001

15. Van Velthoven V, Reinacher PC, Klisch J, Neumann HP, Gläsker S: Treatment of intramedullary hemangioblastomas, with special attention to von Hippel-Lindau disease. Neurosurgery 53:1306-1314, 2003

16. Wang C, Zhang J, Liu A, Sun B: Surgical management of medullary hemangioblastoma. Report of 47 cases. Surg Neurol 56:218-227, 2001

\section{Author Contributions}

Conception and design: Joaquim, Ghizoni, Tedeschi. Acquisition of data: Joaquim, da Silva, Tedeschi. Analysis and interpretation of data: Joaquim, Ghizoni, da Silva, Tedeschi. Drafting the article: Joaquim, Ghizoni, dos Santos, Valadares, Tedeschi. Critically revising the article: Joaquim, Ghizoni, dos Santos, Valadares, Tedeschi. Reviewed submitted version of manuscript: Joaquim, Ghizoni, dos Santos, Valadares, Tedeschi. Approved the final version of the manuscript on behalf of all authors: Joaquim. Statistical analysis: Joaquim. Administrative/technical/material support: Joaquim, Tedeschi. Study supervision: Joaquim, Tedeschi.

\section{Correspondence}

Andrei F. Joaquim, Rua Antônio Lapa 280, S 506, Cambuí, Campinas-SP 13025-240, Brazil. email: andjoaquim@yahoo.com. 